\title{
Secure Watermarking Scheme for Color DICOM Images in Telemedicine Applications
}

\author{
Kamred Udham Singh ${ }^{1}$, Hatem Salem Abu-Hamatta ${ }^{2}$, Abhishek Kumar ${ }^{3}$, Achintya Singhal ${ }^{4}$, \\ Mamoon Rashid ${ }^{5, *}$ and A. K. Bashir ${ }^{6}$ \\ ${ }^{1}$ Department of Computer Science and Information Engineering, National Cheng Kung University, Tainan, Taiwan \\ ${ }^{2}$ Department of Applied Sciences, Aqaba University College, Al Balqa Applied University, Aqaba, Jordan \\ ${ }^{3}$ School of Computer Science \& IT, JAIN (to be Deemed University), Bangalore, India \\ ${ }^{4}$ Department of Computer Science, Banaras Hindu University, Varanasi, India \\ ${ }^{5}$ Department of Computer Engineering, Faculty of Science and Technology, Vishwakarma University, Pune, 411048, India \\ ${ }^{6}$ Department of Computing and Mathematics, Manchester Metropolitan University, UK \\ *Corresponding Author: Mamoon Rashid. Email: mamoon873@gmail.com \\ Received: 09 April 2021; Accepted: 03 June 2021
}

\begin{abstract}
Teleradiology plays a vital role in the medical field, which permits transmitting medical and imaging data over a communication network. It ensures data reliability and provides convenient communication for clinical interpretation and diagnostic purposes. The transmission of this medical data over a network raises the problems of legal, ethical issues, privacy, and copyright authenticity. The copyright protection of medical images is a significant issue in the medical field. Watermarking schemes are used to address these issues. A gray-level or binary image is used as a watermark frequently in color image watermarking schemes. In this paper, the authors propose a novel non-blind medical image watermarking scheme based on 2-D Lifting Wavelet Transform (LWT), Multiresolution Singular Value Decomposition (MSVD), and LU factorization to improve the robustness and authenticity of medical images. In this scheme, multiple color watermarks are embedded into the colored DICOM (Digital Imaging and Communications in Medicine) images obtained from Color Doppler images (DICOM format), and the average results achieved by our proposed scheme is $46.84 \mathrm{db}$ for Peak Signal-to-Noise Ratio (PSNR), $37.46 \mathrm{db}$ for Signal-to-Noise Ratio (SNR), 0.99 for Quality of Image and 0.998 for Normalized Correlation for various image processing attacks. These results make our watermarking technique an ideal candidate for medical image watermarking.
\end{abstract}

Keywords: Digital; imaging; medicine; multiresolution; medical image; watermarking

\section{Introduction}

Teleradiology allows to transmit radiological images for clinical and health care assistance from remote areas, enabling patients to get a second opinion from specialists worldwide. It also

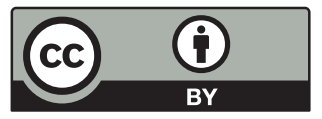

This work is licensed under a Creative Commons Attribution 4.0 International License, which permits unrestricted use, distribution, and reproduction in any medium, provided the original work is properly cited. 
offers the means for healthcare assistance when patients afar from their doctors [1]. Teleradiology can be vital in continuously monitoring the health progress of a patient. Rapid growth and proliferation of computer-assisted technologies in medical sciences, coupled with fast, sophisticated, solid information and communication systems, have exponentially increased medical-related communication over networks [2,3]. Advance medical imaging technologies, viz. MRI, Neuroimaging Informatics Technology Initiative, Color Doppler Imaging, Positron Emission Tomography (PET), CT Scan, etc., have transformed the modern healthcare sector. Due to high printing costs and strenuous communication to distant places, film-based imaging has been replaced by filmless imaging for generating digital images on different devices.

Most hospitals have bulks of patients' data like disease reports, patient's personal information, and medical images in the present scenario. Though hospitals, doctors, radiologist, and providers of medical images are not ready to distribute their patient's medical information in a networked environment due to the copying, ease of interception, unauthorized distribution, and manipulation of the data leading to copyright violations and challenging the integrity of medical data [4], but the need of the hour is that these records be transmitted in low cost and rapid ways through these data communication networks, without losing their qualities and integrity.

Medical information of a patient over the internet is subjected to the bi-fold challenge of noise attacks and damaged by intruders. Thus teleradiology has to ensure reliable data transmission and data authentication of these medical data over such unsecured networks. It also needs to deploy authentication mechanisms like watermarking to secure and authenticate medical images generated using Color Doppler Imaging, X-Ray, PET, MRI, CT Scans, and Neuroimaging Informatics Technology Initiative, etc. Watermarking of digital images provides a powerful way to authenticate the medical data for copyright ownership [5], which is a particular type of image steganography and that can embed the watermark in a carrier image without degrading the quality of the image; thus, preserving the medical image quality and information [6]. Robustness and security of watermarking schemes against various image-processing attacks are among the main challenges in watermarking medical images.

Most state-of-the-art works in the domain of medical image watermarking were proposed for .bmp and .jpg medical images [7]. Some researchers suggested DICOM image watermarking, which was limited to either gray-scale watermarks or gray-scale DICOM images [8]. But in the present medical scenario, various advanced medical imaging technologies have been developed. Like Color Doppler, Ultrasound, etc., these imaging technologies generate full-featured color DICOM medical images instead of flat and featureless .bmp or .jpg images. So the medical healthcare system needs a watermarking scheme for the authentication of these color DICOM images. To meet this requirement of a watermarking technique for the authentication of the color DICOM images, the authors proposed a secure watermarking system for color DICOM images based on LWT, MSVD, and LU factorization. The proposed watermarking methodology attempts to provide higher security and low complexity for DICOM image watermarking, thus overcoming the limitations of the highlighted state-of-art contributions in subsequent sections.

Based on the challenges referred to above, we highlight the significant contributions of this paper as:

(1) A novel non-blind medical image watermarking scheme is developed based on 2-D Lifting Wavelet Transform (LWT), Multiresolution Singular Value Decomposition (MSVD), and LU factorization to improve the robustness and authenticity of medical images. 
(2) An efficient watermarking scheme for color DICOM images in securing access over insecure networks is implemented.

(3) Multiple color watermarks are embedded into the colored Digital Imaging and Communications in Medicine (DICOM) image and then extracted successfully from it even after various attacks.

(4) The evaluation of the watermarking scheme is performed on Color Doppler images (DICOM format), and the average results achieved by our proposed system outperform state-of-the-art.

This research article is structured as follows: Section 2 presents the literature survey. Section 3 reviews the MSVD, LWT and LU. Section 4 presents a proposed watermarking scheme. Experimental findings and evaluation of proposed work with medical images are described in Section 5. The robustness analysis of the introduced scheme is discussed in Section 6. Finally, the findings are concluded in Section 7.

\section{Related Works}

The rapid growth of telemedicine motivated researchers to work on medical imaging. Medical images are broadly classified as grayscale and color images. Moreover, the medical images are often grayscale, having lower faint contrast and faint characteristics due to the conditional limit. Therefore, it is hard for doctors to diagnose and interpret grayscale medical images [9]. Hence, researchers pay more emphasis to color images because of their numerous applications in the era of radiology.

In the present scenario, most medical organizations use color DICOM images for better diagnosing and color trademarks (watermark or logo) for greater security and authenticity. Since DICOM image is augmented with sensitive metadata, copyright protection of the Color Doppler Image, MRI, CT Scan, etc. became a more crucial problem. Consequently, it is necessary to develop a watermarking technique for color medical images with holographic logos. Watermarking methods are classified broadly into two types, viz. spatial domain and transform domain (frequency domain). In the spatial domain image, watermarking technique data is directly embedded in a host image by modifying its bits. In the transform domain or frequency domain, the watermarking image is transformed from spatial domain to frequency domain using various transformations such as Discrete Cosine Transform (DCT), Discrete Fourier Transform (DFT), and Discrete Wavelet Transform (DWT). Compared to the spatial domain, transform domain watermarking techniques are computationally complex, but these techniques provide more embedding capacity and are more robust against noise attack [10]. In the last few years, the researchers developed many watermarking methods for the color image, based on a different type of matrix decomposition.

In the literature, several techniques are there for image watermarking based on various matrix decompositions. Onur et al. [11] proposed a scheme based on a hybrid image-watermarking scheme on DWT, SVD, Arnold's Cat Map, and LU matrix decomposition. The author initially applied the DWT on the host image, which decomposes it into four frequency sub-bands. It then uses the LU factorization (LDU) on the LL band that decomposes it in L, D, and $U$ and then applies the SVD on the D component. D diagonal coefficients of singular value are modified with the oblique factors of the unique value's chaotic mapped watermark. Dongyan et al. [12] proposed a watermarking scheme for color images that work on LU, DWT, and Arnold Transform. The author initially applies the DWT on the host image and gets the four sub-band as usual. Now the 
author applies the LU factorization on the HL and LH bands. Furthermore, Arnold transform applies to the watermark, and then bits of a watermark are embedded using the quantization method. Gaurav et al. [13] suggested a semi-blind watermarking scheme and inserted a grayscale watermark in the host image. In this scheme, the author applied DWT on the host image and created a reference image from all sub-band. After that, the authors applied the SVD on the reference image and watermark. The watermark insertion is achieved by using a reference image's singular values with the watermark's singular values $\mathrm{Z}$.

Zhiqiu et al. [14] proposed a robust null-watermarking scheme based on quaternion polar harmonic Fourier and chaotic map. This scheme is more robust against geometric and noise attacks. Onur et al. [15] suggested that the hybrid watermarking system that relies on DWT \& SVD applies the DWT to the host picture first and then the SVD to the LL band. Now the author embedded the watermark in the diagonal singular value by modifying it. Saeid et al. [16] propose a hybrid scheme. The authors initially applied DWT on the host image, partitioned the LH and HL into $8 \times 8$ blocks, and then apply the DCT on these blocks. After that author, apply the SVD on those blocks. Singular SVD values can be used to match the watermark.

Falgun et al. [17] recently proposed a blind image-watermarking scheme focused on the discrete transformation of the value's wavelet and singular decomposition. Within this scheme, the author initially applies DWT to the region of interest of medical images to obtain specific frequency sub-bands of its wavelet decomposition. The author applied SVD on the lower frequency sub-band (LL band) of the region of interest to get different singular matrices. Watermark's bits are embedded in these pairs by modifying them using a certain threshold. This scheme is extended to different types of medical images, viz. CT scan, mammography, and X-ray. In the article [18], Singh et al. introduced a color image watermarking scheme that uses SVD, DWT, and QR Code in which QR code is used as the image of a watermark. The author initially applies the DWT on the host image and again applies it to the lower frequency band. Then divide the range of frequencies into a block size of $\mathrm{m} * \mathrm{n}$, then locate each block's RGB component to use the SVD to each RGB part and get unique watermarking values.

Xiyao et al. [19] proposed a hybrid scheme based on recursive dither modulation, Slantlet transform, and SVD decomposition. They stated that the scheme is more subtle and robust against attacks. In the article [20], Gao et al. suggested an LWT-derived reversible-image watermarking scheme and chaotic system. The author initially divided the host image into non-overlapping blocks and then applied the LWT on each block and embedded the watermark into every block's LL band. The chaotic system is used for the shuffling of the position of blocks. This scheme is robust towards the cropping attack. Choudhary et al. [7] presented a passive image watermarking scheme using Polar Cosine Transform (PCT). The author initially extracts the image feature using PCT and then applied the patch match algorithm for forge regions to embed the watermark. Souvik et al. [21] present a strategy focused on LWT and SVD. The author first applies the LWT to the host image and divides the LL band into 2X2 size blocks. Furthermore, they used the SVD on these blocks and embedded the binary watermark by modifying these blocks' singular values. In the article [22], Singh et al. suggested a new system based on LWT. In this scheme author initially apply the three-level LWT and divides the LH sub-band into non-overlapped blocks, and these blocks are randomly shuffled. A binary watermark is embedded in these blocks. The main drawback of this system is that it cannot withstand some attacks, i.e., filtering rotational attacks on salt and pepper noise. Singh et al. [23] proposed another scheme in which the three-level LWT was initially applied to the host image, and the binary watermark was embedded in the HL. 
After reviewing the literature in-depth and their contributions, we found little state-of-the-art contributions are made for the color watermarking DICOM images. Recognizing the importance of color DICOM imaging to deter the challenges in transmitting over an unsecured network, a watermarking scheme, especially for color DICOM images generated by various latest medical equipment like Color Doppler, ultrasound, etc is proposed. In this scheme, multiple color watermarks are inserted in DICOM images instead of a single watermark without degrading the original image's quality. It is a secure hybrid watermarking scheme based on the LWT, MSVD, and LU factorization and robust against the various image processing attacks.

\section{Preliminaries}

This section describes the methodology used in this research. For secure image watermarking, we have used Multiresolution Singular Value Decomposition (MSVD), LU Factorization and, Lifting Wavelet Transform (LWT) to locate the most impactful feature of the color DICOM image and subsequently secure it using this Image Watermarking Scheme for Telemedicine Applications.

\subsection{Multiresolution Singular Value Decomposition (MSVD)}

MSVD computational process is simple and easily can be used in real-time applications. Like Discrete Cosine Transform (DCT), Fast Fourier Transform (FFT), and Wavelet, it doesn't have a fixed set based on vectors. One resolution feature is easy to detect, but the characteristics of multiresolution techniques are undetectable. It is the replacement of the wavelets and is comparable to the Wavelet Multi-Resolution Analysis (MRA) because it decomposes the original image into different frequency bands (lower frequency and higher frequency LL, LH, HL, and HH) [24]. MSVD has low computational power than wavelet transformation and directional information such as wavelet transformations. In MSVD, decomposition is done based on the data set [25]. It can be used at multiple levels and often provide robustness against the various geometrical image processing attack viz. rotation, cropping, and resizing.

For a matrix, A having dimension $\mathrm{M} * \mathrm{~N}$, the singular value decomposition (SVD) is the factorization of A into the three-matrix product and is shown in Eq. (1) as:

$A=U S V^{T}$

where $\mathrm{V}$ and $\mathrm{U}$ columns are orthonormal, and matrix $\mathrm{S}$ is a diagonal matrix in descending order of singular values.

MSVD for image (I) of size $\mathrm{M} \times \mathrm{N}$ is computed by initially reshaping $\mathrm{I}$ to a matrix $\mathrm{I}^{\prime}$ of size $4 \times \mathrm{MN} / 4$. Consequently, the singular value decomposition of $\mathrm{I}^{\prime}$ is shown in Eq. (2) as:

$[\mathrm{U}, \mathrm{S}]=\operatorname{SVD}\left(\mathrm{I}^{\prime}\right)$

Now, matrix $\mathrm{T}$ is shown by Eq. (3) as:

$T=V^{T} \mathrm{I}^{\prime}$

where matrix $U$ of size $4 \times 4$, matrix $\mathrm{S}$ of size $4 \times \mathrm{MN} / 4$ and matrix $\mathrm{T}$ of size [ $4 \times \mathrm{MN} / 4]$. In matrix $T$ rows I, II, III, and IV are reshaped to produce size matrices $M / 2 \times M / 2$. Such reformatted matrices are the image sub-bands i.e., LH, HL, LL, HH. The LL band includes the lower frequency information, and the high-frequency information is found in other sub-bands i.e., HL, LH, and HH. In this paper, we use LH band for watermarking. 


\subsection{Theorem: (LU Factorization)}

If $\mathrm{A} \in \mathrm{Rnxn}$ and $\operatorname{det}(\mathrm{A}(1: \mathrm{k}, 1: \mathrm{k})) \neq 0$ for $\mathrm{k}=1: \mathrm{n}-1$, instead there is a triangular lower unit $\mathrm{L} \in \mathrm{Rnxn}$ and a triangular upper unit $\mathrm{U} \in \mathrm{Rnxn}$ such that $\mathrm{A}=\mathrm{LU}$. If this is the scenario, and $A$ is non-singular, the variables are special and $\operatorname{det}(A)=u_{11}, \ldots, u_{n n}[26]$.

LU factorization is an algebraic "high-level" definition of Gaussian elimination. Triangular system solving is an easy $\mathrm{O}(\mathrm{n} 2)$ computation. Any square matrix $\mathrm{A}$ is writable as a product of matrices L and U. LU for all instance's decomposition may not exist. Theorem 1 specifies the conditions in which the decomposition can be carried out. LU decomposition of $3 \times 3$ matrix is depicted in Eq. (4) as:

$$
\begin{aligned}
\mathrm{A}= & {\left[\mathrm{a}_{1}, \mathrm{a}_{2}, \mathrm{a}_{3}\right]=\left[\begin{array}{lll}
\mathrm{a}_{11} & \mathrm{a}_{12} & \mathrm{a}_{13} \\
\mathrm{a}_{21} & \mathrm{a}_{22} & \mathrm{a}_{24} \\
\mathrm{a}_{31} & \mathrm{a}_{32} & \mathrm{a}_{34}
\end{array}\right] } \\
& \text { Any matrix } A=\left[\begin{array}{lll}
a_{11} & a_{12} & a_{13} \\
a_{21} & a_{22} & a_{24} \\
a_{31} & a_{32} & a_{34}
\end{array}\right] \text { can be factorized in } \\
L= & {\left[\begin{array}{lll}
1 & 0 & 0 \\
a_{21} & 1 & 0 \\
a_{31} & a_{32} & 1
\end{array}\right], \quad U=\left[\begin{array}{lll}
a_{11} & a_{12} & a_{13} \\
0 & a_{22} & a_{24} \\
0 & 0 & a_{34}
\end{array}\right] . }
\end{aligned}
$$

where L stands for lower-triangular matrix, which has diagonal Identity Elements and upper nondiagonal elements are zero. $\mathrm{U}$ is the upper triangular matrix with some coefficients on the lower diagonal non-diagonal components, zero. The inverse decomposition of the LU can be defined as

$\mathbf{L} \times \mathbf{U}=\mathbf{A}$

The decomposition of the LU matrix has less computational complexity than the SVD and QR [27,28] and decomposition with LU is an interim step in the decomposition of QR. In factorization operation, count of $\mathrm{LU}$ is $\frac{2}{3} \boldsymbol{m}^{2}$ whereas for QR factorization the operation count is $\frac{4}{3} \boldsymbol{m}^{3}$, where $\mathrm{m}$ is the size of the matrix [22]. Due to lower computational complexity, LU will be explored for the watermarking technique.

\subsection{Lifting Wavelet Transform (LWT)}

In the article, Jayant et al. [29] suggested LWT. The essential characteristic of this scheme is that the spatial domain arises from all the constructions. It overcomes the conventional wavelet transformation limitations by decreasing the complexity of time and space (because it does not require complex mathematical calculations, unlike traditional wavelet transform). So computing wavelet transforms an efficient and most straightforward algorithm. Lifting Scheme does not rely on transforms from Fourier. Digital signals are a sequence of integers, but the resultant message is a sequence of floating-point numbers after the wavelet transform. This hinders the 
possibility of a perfect or a reasonable reconstruction of the signal. A transformation algorithm should have a feasible reversible implementation. It preserves the messages even after the transform, i.e., it behaves as an operator, which transforms integer-based sequences again into integer-based sequence signals. Lifting scheme provides this facility of preserving the domain of transform (i.e., integer to integer) with better computational efficiency than the conventional wavelet transformations, making it a commonly used change in image processing [30].

LWT decomposes the image into four frequency subgroups viz. LH, HL, LL, and HH. The sub-band LL has a lower frequency that contains most of the picture's energy, and other subbands ( $\mathrm{LH}, \mathrm{HL}$, and $\mathrm{HH}$ ) have elements of higher frequency that contain lower energy in the picture. The LWT can be applied several times on the LL band to decompose into sub-bands because it includes most original image energy.

\section{Proposed Scheme}

DICOM images are widely used in medical imaging, as they contain high-definition images and patient metadata, often known as the Electronic Patient Record (EPR). Watermarking of DICOM images is a crucial task in comparison to the other image formats viz .jpg, .bmp, .tiff, .png etc. The watermarking scheme must be imperceptible. There must not be any noticeable change in color, hue, and intensity, which may hamper the image's diagnosis. The metadata of the DICOM image is highly sensitive, so it has to be preserved carefully. This paper suggests a new watermarking algorithm for color DICOM images based on MSVD, LWT, and LU factorization, including the watermark embedding and extraction processes.

Table 1: Watermark embedding algorithm

\begin{tabular}{ll}
\hline Step & Explanation \\
\hline 1. & Extract patient information from the DICOM image. \\
2. & Disintegrate host and watermarked image into RGB components. \\
3. & Apply the LU factorization on red, green and blue watermarked components. \\
4. & Perform MSVD on RGB components of host-image that disintegrate it into four \\
frequencies sub-bands LH, HL, LL, and HH. \\
Perform LWT on HL band of MSVD that also disintegrates it into four sub bands LH, \\
HL, LL, and HH. \\
The factorized watermark components are embedded into respective transformed \\
component of the host image by selecting the LL band of it, which includes most of \\
the host-image energy. Watermark is augmented in the image as below: \\
$\quad \boldsymbol{x}^{\prime}=\boldsymbol{x}+\boldsymbol{\alpha} \boldsymbol{y}$ \\
where $\boldsymbol{x}^{\prime}$ is the transformed component of the watermark image, y is the factorized \\
watermark component, x is the transform image of host image and $\boldsymbol{\alpha}$ is the embedding \\
strength. \\
Perform every component's inverse LWT. \\
Perform inverse MSVD on each final watermarked image portion. \\
Embedded patient information in watermark image. \\
9.
\end{tabular}




\subsection{Insertion Algorithm}

The metadata of the DICOM image is extremely sensitive, so we initially isolate this metadata from the DICOM image. Now medical images and watermarks are decomposed into RGB components, and each color component is subjected to the MSVD that decomposes it into four frequency bands viz. LH, HL LL, and HH. HL bands contain the most positive values, so we apply the LWT on the HL band, which decomposes into four different sub-band frequency, viz. LL, LH, HL, and HH. Most of the information on the images contained in LL band. Therefore, we select the LL band of the watermark embedding. Now apply the LU factorization on the watermark's primary color component, generate the new vector, and embed these vectors into the LL band of the LWT. Watermark embedding is controlled via a scaling factor (alpha) and performs the inverse process to generate the image. Finally, we integrate the patient's metadata with this watermarked image and create the watermarked DICOM image. The watermark embedding algorithm is explained in Tab. 1 and is presented in Fig. 1.

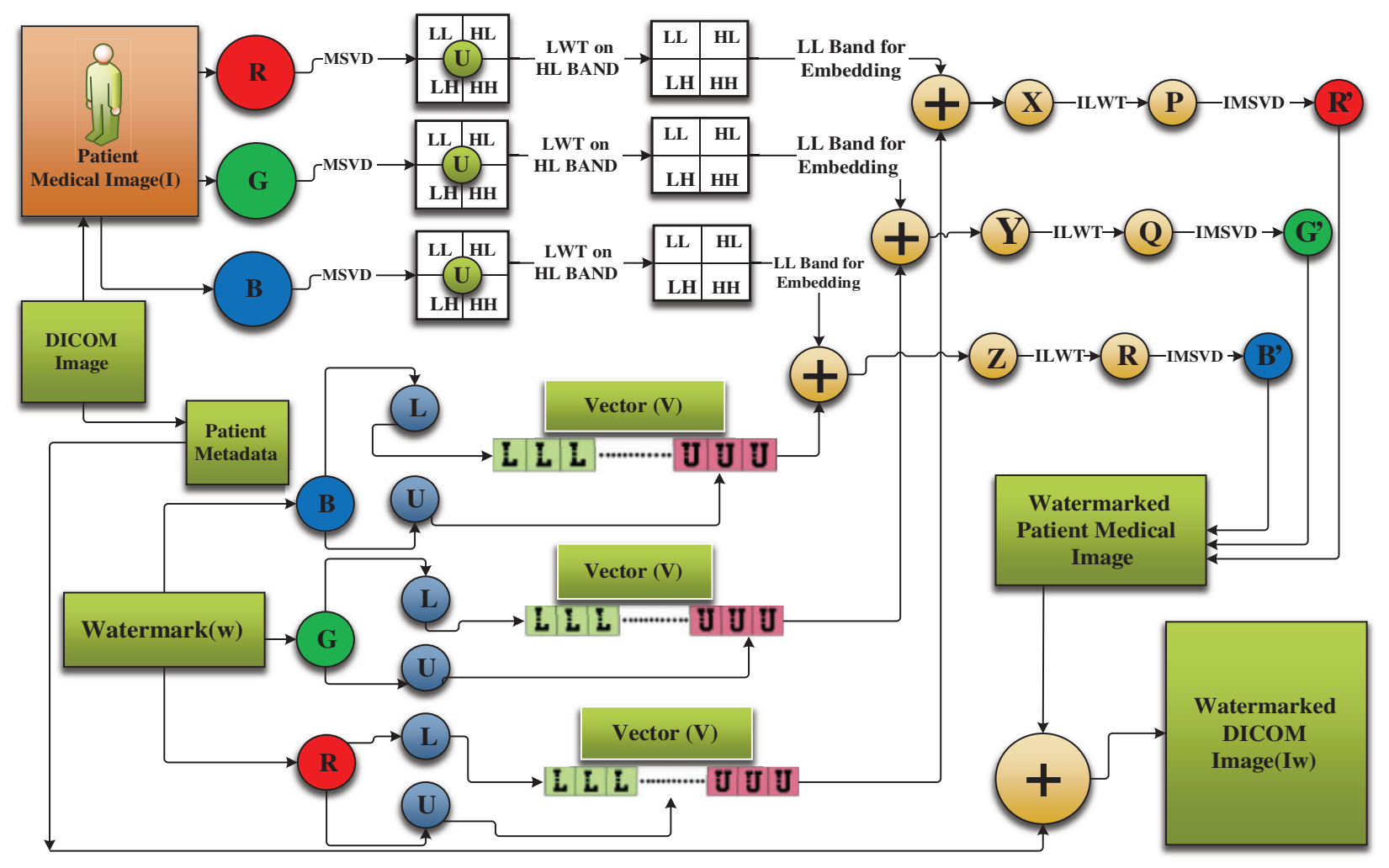

Figure 1: Watermark insertion process

\subsection{Extraction Algorithm}

The extraction of watermarks is an integral part of the success of any watermarking scheme. In Fig. 2, the watermark extraction method for the suggested scheme is illustrated. As shown in Fig. 2, the actual DICOM image is required when the watermark is extracted from the watermarked DICOM image. Initially, metadata is isolated from original and watermarked DICOM 
images and decomposed into three primary color components (RGB). After that, apply the MSVD on each primary color component of the original and watermarked image. Then HL band of MSVD is subjected to wavelet transformation that decomposes both images in four sub-bands with various frequencies, i.e., HL, LH, LL and, HH. Using LL sub-bands of both images, we separated the noisy components from the watermarked DICOM image. Then perform the Inverse LU factorization on these noise components to regenerate the watermarks. The extraction method algorithm is portrayed in Fig. 2 and explained in Tab. 2.

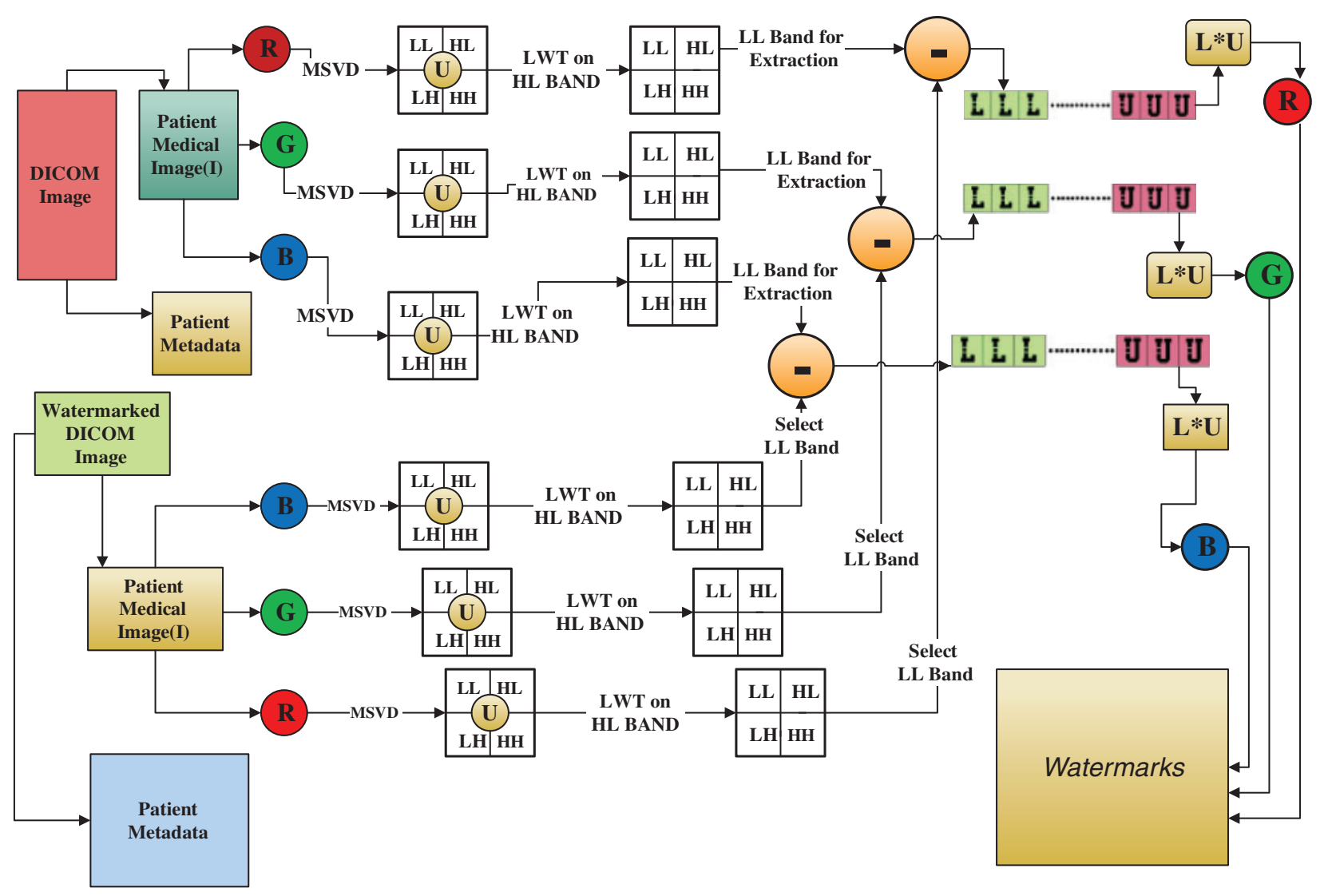

Figure 2: Watermark extraction process

\section{Results and Discussion}

The watermarking scheme trial was performed over color DICOM images (primary data) generated by the Color Doppler test, congregated from S.S.L. Hospital Banaras Hindu University Varanasi India (primary source), under ethical medical practices, without revealing the patient's identity and only for the experimental purpose. The scheme was implemented on MATLAB platform configured on Intel core TM i7-5500U CPU $2.5 \mathrm{GHz}$ processor with $8 \mathrm{~GB}$ RAM and running Windows10 operating system. The host color DICOM images of dimension $1024 \times 768$ pixels, the BMP format watermark image of dimension $32 \times 32$ pixels, and bit depth of 24 bits are used in the experiment. 
Table 2: Watermark extraction algorithm

\begin{tabular}{|c|c|}
\hline Step & Explanation \\
\hline 1. & Use DICOM image to retrieve patient records from the host and watermarked image. \\
\hline 2. & Decompose host image into RGB parts. \\
\hline 3. & Transform watermarked image into RGB parts. \\
\hline 4. & $\begin{array}{l}\text { Perform MSVD on watermarked image and host image to obtain HL LH, LL, and HH } \\
\text { sub-bands. }\end{array}$ \\
\hline 5. & Perform LWT on the HL sub band on obtained watermarked and host image. \\
\hline \multirow[t]{7}{*}{6.} & $\begin{array}{l}\text { Obtain watermark from watermarked image by performing reversal of embedded steps } \\
\text { using original image }\end{array}$ \\
\hline & $y=\left(I^{+}-I\right) / \alpha$ \\
\hline & where \\
\hline & $I^{\prime}$ is the watermarked image. \\
\hline & $y$ is the factorized watermark component. \\
\hline & $I$ is the original image \\
\hline & $\alpha$ is the embedding strength. \\
\hline 7. & Perform inverse LU factorization on extracted components. \\
\hline 8. & Concatenate the components and obtain the extracted watermark. \\
\hline
\end{tabular}

\subsection{Performance Measures}

The imperceptibility of the watermarking scheme is tested on various datasets and projected in this paper on six different DICOM images. The outcomes are inspected regarding image quality measurements viz. PSNR, MSE, Universal Image Quality Index, and Normalized Correlation. PSNR and two-dimensional Normalized Correlation (NC) is utilized as a measure for perceptual imperceptibility. For the watermark perceptibility estimation, PSNR is deliberated which, is outlined in terms of Eqs. (5)-(7) as follows [31]:

$M S E($ Mean Square Error $)=\frac{1}{m * n} \sum_{i=0}^{m} \sum_{j=0}^{n}\left(A_{i j}-B_{i j}\right)^{2}$

$\operatorname{PSNR}($ Peak Signal to Noise Ratio $)=10 * \log _{10} \frac{(M a x)^{2}}{\frac{1}{m * n} \sum_{i=0}^{m} \sum_{j=0}^{n}\left(A_{i j}-B_{i j}\right)^{2}}$

$N R=10 * \log _{10} \frac{\sum_{i=1}^{n} \sum_{j=1}^{m}\left(A_{i j}\right)^{2}}{\sum_{i=1}^{n} \sum_{j=1}^{m}\left(A_{i j}-B_{i j}\right)^{2}}$

The watermarking system's robustness is calculated using Normalized correlation (NC) [32,33] and, between the original watermark $\mathrm{W}$ and the extracted watermark $\mathrm{W}+$, which is defined in terms of Eq. (8).

$$
N C=\frac{\sum_{c=1}^{3} \sum_{x=1}^{p} \sum_{y=1}^{q}\left(W(x, y, c) \times W^{+}(x, y, c)\right)}{\sqrt{\sum_{c=1}^{3} \sum_{x=1}^{p} \sum_{y=1}^{q}\left(W(x, y, c)^{2}\right)} \sqrt{\sum_{c=1}^{3} \sum_{x=1}^{p} \sum_{y=1}^{q}\left(W^{+}(x, y, c)^{2}\right)}}
$$


Universal image quality index $\mathbf{Q}[34]$ is defined in terms of Eq. (9) as follows:

$Q=\frac{4 \sigma_{x y} \bar{x} \bar{y}}{\left(\sigma_{x}^{2}+\sigma_{y}^{2}\right)\left[(\bar{x})^{2}+(\bar{y})^{2}\right]}$

where $\bar{x}, \bar{y}, \sigma_{x}^{2}$ and $\sigma_{y}^{2}$ are defined interms of Eq. (10) as follows:

$\bar{x}=\frac{1}{N} \sum_{i=1}^{N} x_{i} \quad$ and $\quad \bar{y}=\frac{1}{N} \sum_{i=1}^{N} y_{i}$

$\sigma_{x}^{2}=\frac{1}{N-1} \sum_{i=1}^{N}\left(x_{i}-\bar{x}\right)^{2} \quad$ and $\quad \sigma_{y}^{2}=\frac{1}{N-1} \sum_{i=1}^{N}\left(y_{i}-\bar{y}\right)^{2}$

$\sigma_{x y}=\frac{1}{N-1} \sum_{i=1}^{N}\left(x_{i}-\bar{x}\right)\left(y_{i}-\bar{y}\right)$

where $X=\left\{x_{i} \mid i=1,2,3, \ldots, N\right\}$ and $Y=\left\{y_{i} \mid i=1,2,3, \ldots, N\right\} . \mathrm{X}$ and $\mathrm{Y}$ are the original and the test image signals, respectively.

Universal image quality index $\mathrm{Q}$ is the product of three components that can be defined in terms of Eq. (11) as:

$Q=\frac{\sigma_{x y}}{\sigma_{x} \sigma_{y}} \times \frac{2 \bar{x} \bar{y}}{(\bar{x})^{2}+(\bar{y})^{2}} \times \frac{2 \sigma_{x} \sigma_{y}}{\sigma_{x}^{2}+\sigma_{y}^{2}}$

\subsection{Analysis of Proposed Scheme}

The trial of the proposed scheme is presented on six diverse DICOM images viz. Img1, Img2, Img3, Img4, Img5, and Img6, depicted in Fig. 3, determine and analyze the imperceptibility and robustness parameters. The metadata containing the affected person's information is also preserved within the watermarked DICOM image. The scheme inserted various copies of the watermark across color DICOM images, and consequently, multiple watermarks are extracted from the watermarked DICOM image. All the images used for the experiment are of the same dimension and from the same source, which results in the same number of inserted watermarks. Consequently, we tend to determine that 12 watermarks were embedded throughout all the images, and therefore, a similar number of watermarks are extracted. Tab. 3 demonstrates the performance measures of watermarked images from Img1 to Img6.

Both Quality parameters NC and Q have values close to one, indicating a virtually excellent correlation between the initial and watermarked images. PSNR of the watermarked image is over thirty-nine decibels, which suggests that the watermarked image quality is exceptionally considerable.

\subsection{Analysis of the Proposed Scheme for Different Number of Watermarks Insertion}

This scheme embeds multiple watermarks in the host image. The maximum watermark insertions in the DICOM image without influencing the diagnosis based on a radiologist's watermarked DICOM image are demonstrated in Tab. 4. The scheme is further tested with many watermark insertions and found that the outcomes are promising. 


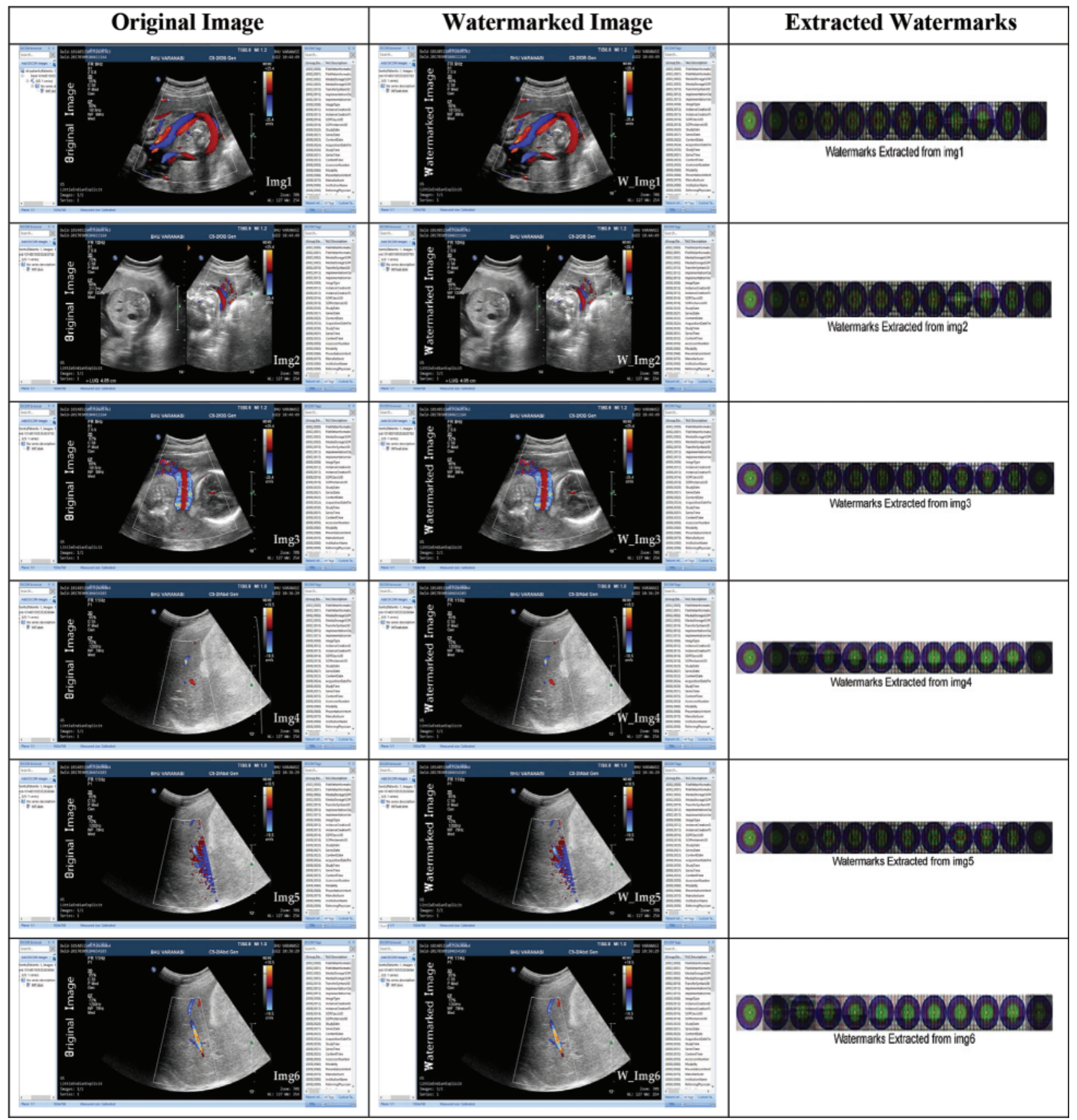

Figure 3: Test DICOM images with their corresponding watermarked images and extracted watermarks

Tab. 4 demonstrates that the quality metrics are directly proportionate to the watermark insertion, and no notible changes are observed. Nevertheless, if we evaluate the PSNR of the img1 at 12 watermark insertions \& 2 watermark insertions, there is a significant variation from 46.98049 to $54.356909 \mathrm{db}$. But there are no significant variations in NC and Q; that's why not 
influencing the diagnosis. The results of the suggested technique are compared with other existing techniques [17,34] in Tab. 5, and it is observed that the results are more significant.

Table 3: Qualitative analysis of watermarked images

\begin{tabular}{lllllll}
\hline $\begin{array}{l}\text { Medical } \\
\text { images }\end{array}$ & $\begin{array}{l}\text { NC of } \\
\text { image }\end{array}$ & $\begin{array}{l}\text { Q of } \\
\text { image }\end{array}$ & $\begin{array}{l}\text { PSNR } \\
\text { of image }\end{array}$ & $\begin{array}{l}\text { SNR of } \\
\text { image }\end{array}$ & $\begin{array}{l}\text { MSE of } \\
\text { image }\end{array}$ & $\begin{array}{l}\text { No of } \\
\text { watermarks }\end{array}$ \\
\hline img1 & 0.998893 & 0.999794 & 46.98049 & 35.99315 & $2.00 \mathrm{E}-05$ & 12 \\
img2 & 0.999184 & 0.999841 & 46.37763 & 38.12030 & $2.30 \mathrm{E}-05$ & 12 \\
img3 & 0.998919 & 0.999812 & 46.94200 & 36.82462 & $2.02 \mathrm{E}-05$ & 12 \\
img4 & 0.998744 & 0.999748 & 46.88058 & 38.41127 & $2.05 \mathrm{E}-05$ & 12 \\
img5 & 0.998716 & 0.999741 & 46.91947 & 37.77665 & $2.03 \mathrm{E}-05$ & 12 \\
img6 & 0.998719 & 0.999743 & 46.91323 & 37.64021 & $2.04 \mathrm{E}-05$ & 12 \\
\hline
\end{tabular}

Table 4: Performance verification

\begin{tabular}{llllllll}
\hline No. of watermarks & Parameter & Img1 & Img2 & Img3 & Img4 & Img5 & Img6 \\
\hline 12 & NC & 0.998893 & 0.999184 & 0.998919 & 0.998744 & 0.998716 & 0.998719 \\
& Q & 0.999794 & 0.999841 & 0.999812 & 0.999748 & 0.999741 & 0.999743 \\
& PSNR & 46.98049 & 46.37763 & 46.942 & 46.88058 & 46.91947 & 46.91323 \\
& SNR & 35.99315 & 38.1203 & 36.82462 & 38.41127 & 37.77665 & 37.64021 \\
8 & NC & 0.99936 & 0.999535 & 0.999373 & 0.99929 & 0.999273 & 0.999276 \\
& Q & 0.999856 & 0.999888 & 0.999869 & 0.999828 & 0.999824 & 0.999825 \\
& PSNR & 48.57695 & 47.88096 & 48.54545 & 48.48187 & 48.54336 & 48.53221 \\
& SNR & 37.58961 & 39.62362 & 38.42808 & 40.01256 & 39.40053 & 39.25919 \\
6 & NC & 0.99948 & 0.999638 & 0.9995 & 0.999434 & 0.999425 & 0.999424 \\
& Q & 0.999899 & 0.999924 & 0.999908 & 0.999877 & 0.999874 & 0.999874 \\
& PSNR & 49.73456 & 49.39451 & 49.73549 & 49.64057 & 49.65886 & 49.66389 \\
& SNR & 38.74722 & 41.13717 & 39.61811 & 41.17126 & 40.38584 & 40.52107 \\
& NC & 0.999689 & 0.999761 & 0.999695 & 0.99961 & 0.9996 & 0.999601 \\
& Q & 0.999922 & 0.99994 & 0.999929 & 0.999907 & 0.999905 & 0.999905 \\
& PSNR & 51.42444 & 50.7233 & 51.3892 & 51.33956 & 51.41552 & 51.40477 \\
& SNR & 40.4371 & 42.46596 & 41.27182 & 42.87025 & 42.2727 & 42.13175 \\
& NC & 0.999465 & 0.999632 & 0.999484 & 0.999437 & 0.999423 & 0.999424 \\
& Q & 0.999901 & 0.999924 & 0.99991 & 0.999882 & 0.99988 & 0.999881 \\
& PSNR & 53.03681 & 52.45908 & 52.98594 & 53.06154 & 53.11815 & 53.11613 \\
& SNR & 42.04947 & 44.20175 & 42.86856 & 44.59223 & 43.97533 & 43.84311 \\
& NC & 0.999856 & 0.999862 & 0.999858 & 0.999855 & 0.999855 & 0.999855 \\
2 & Q & 0.999962 & 0.999972 & 0.999965 & 0.999953 & 0.999952 & 0.999952 \\
& PSNR & 54.35691 & 53.82507 & 54.33934 & 54.33447 & 54.37937 & 54.36589 \\
& SNR & 43.36957 & 45.56773 & 44.22196 & 45.86516 & 45.23655 & 45.09287 \\
\hline & & & & & & & \\
& & & & & &
\end{tabular}


Table 5: Performance comparison of proposed and existing scheme

\begin{tabular}{|c|c|c|c|c|}
\hline \multicolumn{2}{|c|}{$\begin{array}{l}\text { Proposed scheme (Two color } \\
\text { watermarks insertion of } 32 \times 32 \\
\text { size) }\end{array}$} & \multicolumn{2}{|c|}{$\begin{array}{l}\text { Falgun et al. (single binary } \\
\text { watermark insertion of } 32 \times 32 \\
\text { size) [17] }\end{array}$} & \multirow{2}{*}{$\begin{array}{l}\text { Agung et al. } \\
\text { PSNR }\end{array}$} \\
\hline $\mathrm{NC}$ & PSNR & $\mathrm{NC}$ & PSNR & \\
\hline 0.999856 & 54.35691 & 1 & 46.8760 & 47.7949 \\
\hline 0.999862 & 53.82507 & 1 & 45.4468 & 47.4966 \\
\hline 0.999858 & 54.33934 & 0.9397 & 44.4853 & 48.5741 \\
\hline 0.999855 & 54.33447 & 1 & 44.7888 & 47.0075 \\
\hline 0.999855 & 54.37937 & 0.9900 & 43.4096 & 47.4085 \\
\hline 0.999855 & 54.36589 & 0.9728 & 41.6728 & 46.8191 \\
\hline
\end{tabular}

\section{Robustness Analysis}

The watermarked color DICOM images have been subjected to different image processing/manipulation like Salt \& Pepper, Poisson, Gaussian, Speckle noise, and image format conversion that may cause watermarks to purge as an attack. Sustenance of the watermarking scheme against these attacks is the test of the robustness of the system. Since the algorithm deals with medical images, we always keep in mind that the diagnosis based on these watermarked images may cause the patient's life and death. Therefore, the deterioration of these watermarked images beyond an acceptable quality is not acceptable. As the proposed scheme is a multiple watermark insertion scheme for increased robustness; hence we analyze the algorithm either with the maximum inserted watermarks or fewer, which i affects the diagnosis. Therefore, the technique is tested with the different attacks having different parameters until the quality of the image is acceptable according to the quality metrics. We have also tested the scheme to convert the DICOM images in flat and featureless formats like .bmp or .jpg. It is common to communicate in these formats because larger DICOM images require a resource-intensive communication medium.

\subsection{Salt and Pepper Noise Attack}

Salt and pepper noise attack implemented with noise density 0.001 and 0.002 on the watermarked DICOM picture, and then the watermark is successfully extracted. Tab. 6 tabulates the parametric data post-attack on watermarked DICOM healthcare images, and the findings reflect that the efficiency of the proposed technique for DICOM images is promising.

Table 6: Salt and pepper noise attack with noise density 0.001 and 0.002 and extracted watermarks Noise density NC of image Q of image PSNR of image SNR of image Extracted watermarks

\begin{tabular}{|c|c|c|c|c|c|}
\hline 0.001 & 0.999611 & 0.996278 & 33.95385 & 22.96636 & 40 \\
\hline 0.002 & 0.99902 & 0.992181 & 30.71863 & 19.73114 & \\
\hline
\end{tabular}




\subsection{Gaussian Noise Attack}

Gaussian noise attack introduced with variance 0.001 to 0.002 on the watermarked DICOM image, and then the watermark is successfully extracted by the suggested watermarking method. Tab. 7 shows the attack parameter and the subsequent image quality metrics. The parametric values are positive performance indicators of the proposed technique for DICOM images.

Table 7: Gaussian noise attack with variance 0.001 and 0.002 and extracted watermarks

\begin{tabular}{llllll}
\hline Variance & NC of image & Q of image & PSNR of image & SNR of image & Extracted watermarks \\
\hline 0.0001 & 1.007018 & 0.997245 & 37.12332 & 26.13583 & \\
0.0002 & 1.006025 & 0.996324 & 35.50706 & 24.51957 & \\
\hline
\end{tabular}

\subsection{Poisson Noise Attack}

Poisson noise attack introduced in the watermarked DICOM image, and then original image is effectively extracted by the suggested watermarking technique. Metric values tabulated in Tab. 8 depict the analysis of the attack for the DICOM medical images. The outcome indicates that the performance of the suggested method for DICOM images is promising.

Table 8: Watermarked image after poisson noise attack and extracted watermarks

\begin{tabular}{lllll}
\hline NC of image & Q of image & PSNR of image & SNR of image & Extracted watermarks \\
\hline 0.99985 & 0.999978 & 56.21901 & 45.23152 & \\
\hline
\end{tabular}

\subsection{Speckle Noise Attack}

Speckle noise attack introduced with variance 0.001 to 0.003 on the watermarked DICOM picture, and then the suggested watermarking scheme successfully extracts the watermark. In Tab. 9, evaluation of the DICOM medical image attack is tabled and the findings show that the output of the proposed DICOM image scheme is strong.

Table 9: Speckle noise attack with variance 0.001 to 0.003 and extracted watermarks

\begin{tabular}{llllll}
\hline Variance & NC of image & Q of image & PSNR of image & SNR of image & Extracted watermarks \\
\hline 0.001 & 0.998434 & 0.999306 & 41.26814 & 30.28065 & \\
0.002 & 0.99764 & 0.998617 & 38.27095 & 27.28346 & \\
0.003 & 0.997069 & 0.997929 & 36.51817 & 25.53068 & \\
\hline
\end{tabular}




\section{Conclusion}

Advancements in medical technologies worldwide gave rapid growth to medical imaging, thus providing digital projections in the various parts of the human body to diagnose the medical conditions for treatment purposes. These devices generate high-quality images of patients and provide an easy insight to understand the situation of patients. Nevertheless, this leads to challenges viz. authentication, theft, enforcement of medical ethics, etc. Thus, the watermarking scheme devised for DICOM images augmented with sensitive metadata using the combination of MSVD, and LWT followed by LU factorization addresses these challenges in medical healthcare. Different color DICOM images (1024 x 768 pixels) (primary data) sourced from the Color Doppler test are watermarked with 24-bit color watermark (32 x 32 pixel) have been tested in the proposed scheme. The parameters NC, Q, SNR, PSNR, and MSE are the basis for analyzing the quality of watermarked images. The tradeoff between image quality and watermark insertions ensures yielding the results of utter significance. Our proposed scheme provides robustness towards various image processing attacks such as Poisson, Salt \& Pepper, Speckle, Gaussian interference, and image format conversion. The attacking parameters were controlled in that it does not affect the quality of the watermarked DICOM to the level that it becomes insignificant for medical purposes. The scheme's final result is compared with several other existing schemes, and we found that the elaborated scheme to be noteworthy. The proposed scheme has high potential and can be extended to other medical imaging formats viz. Neuroimaging Informatics Technology Initiative (Nifti) and Minc.

Funding Statement: The authors received no specific funding for this study.

Conflicts of Interest: The authors declare that they have no conflicts of interest to report regarding the present study.

\section{References}

[1] K. Alavi, H. Al-Ahmad and F. Taher, "Medical images protection and authentication using hybrid DWT-DCT and SHA256-MD5 hash functions," in 2017 24th IEEE Int. Conf. on Electronics, Circuits and Systems, Batumi, Georgia, IEEE, pp. 397-400, 2017.

[2] T. Rohit, S. Borra, V. Dwivedi and K. Borisagar, "An efficient medical image watermarking scheme based on FDCuT-DCT," Engineering Science and Technology, An International Journal, vol. 20, no. 4, pp. 1366-1379, 2017.

[3] T. Sriti, A. K. Singh and S. P. Ghrera, "Chaotic based secure watermarking approach for medical images," Multimedia Tools and Applications, vol. 79, no. 26, pp. 4263-4276, 2020.

[4] Z. Aditi, A. K. Singh and P. Kumar, "A proposed secure multiple watermarking technique based on DWT, DCT and SVD for application in medicine," Multimedia Tools and Applications, vol. 77, no. 4, pp. 4863-4882, 2018.

[5] C. Lakshmi, K. Thenmozhi, J. B. B. Rayappan and R. Amirtharajan, "Encryption and watermarktreated medical image against hacking disease-An immune convention in spatial and frequency domains," Computer Methods and Programs in Biomedicine, vol. 159, pp. 11-21, 2018.

[6] T. Sriti, A. K. Singh andS. P. Ghrera, "Multi-layer security of medical data through watermarking and chaotic encryption for tele-health applications," Multimedia Tools and Applications, vol. 78, no. 3, pp. 3457-3470, 2019.

[7] P. Choudhary, S. H. Om and S. Maheshkar, "Authentication of medical images using passive approach," IET Image Processing, vol. 13, no. 13, pp. 2420-2427, 2019.

[8] S. Malini and R. S. Moni, "Image denoising using multiresolution singular value decomposition transform," Procedia Computer Science, vol. 46, pp. 1708-1715, 2015. 
[9] C. D. Singh, A. K. Singh, B. Kumar and J. P. Saini, "Quantization based multiple medical information watermarking for secure e-health," Multimedia Tools and Applications, vol. 78, no. 4, pp. 3911-3923, 2019.

[10] L. C. Chin and C. C. Tsai, "Digital image watermarking using discrete wavelet transform and singular value decomposition," IEEE Transactions on Instrumentation and Measurement, vol. 59, no. 11, pp. 30603063, 2010.

[11] J. Onur, H. G. İlk and E. Elbaş1, "A secure and robust watermarking algorithm based on the combination of dwt, svd, and lu decomposition with arnold's cat map approach," in 2013 8th Int. Conf. on Electrical and Electronics Engineering, Bursa, Turkey, IEEE, pp. 306-310, 2013.

[12] W. Dongyan, F. Yang and H. Zhang, "Blind color image watermarking based on DWT and LU decomposition," Journal of Information Processing Systems, vol. 12, no. 4, pp. 765-778, 2016.

[13] B. Gaurav and B. Raman, "A new robust reference watermarking scheme based on DWT-SVD," Computer Standards \& Interfaces, vol. 31, no. 5, pp. 1002-1013, 2009.

[14] X. Zhiqiu, X. Wang and C. Wang, "Geometrically invariant color medical image null-watermarking based on precise quaternion polar harmonic Fourier moments," IEEE Access, vol. 7, pp. 122544 $122560,2019$.

[15] J. Onur and E. Elbaşi, "Hybrid non-blind watermarking based on DWT and SVD," Journal of Applied Research and Technology, vol. 12, no. 4, pp. 750-761, 2014.

[16] F. Saeid and M. Moeini, "A robust image watermarking method based on DWT, DCT, and SVD using a new technique for correction of main geometric attacks," Optik, vol. 127, no. 2, pp. 964-972, 2016.

[17] T. N. Falgun and V. K. Srivastava, "A blind medical image watermarking: Dwt-svd based robust and secure approach for telemedicine applications," Multimedia Tools and Applications, vol. 76, no. 3, pp. 3669-3697, 2017.

[18] S. R. Kumar, D. K. Shaw and J. Sahoo, "A secure and robust block based DWT-SVD image watermarking approach," Journal of Information and Optimization Sciences, vol. 38, no. 6, pp. 911-925, 2017.

[19] L. Xiyao, J. Lou, H. Fang, Y. Chen, P. Ouyang et al. "A novel robust reversible watermarking scheme for protecting authenticity and integrity of medical images," IEEE Access, vol. 7, pp. 76580-76598, 2019.

[20] T. G. Gao, T. Gang and Q. L. Gu, "Reversible watermarking algorithm based on wavelet lifting scheme," in Int. Conf. on Wavelet Analysis and Pattern Recognition, Beijing, China, IEEE, vol. 4, pp. 1771-1775, 2007.

[21] S. Souvik and K. Senthilkumar, "A highly secured digital watermarking algorithm for binary watermark using lifting wavelet transform and singular value decomposition," in IET Chennai 3rd International on Sustainable Energy and Intelligent Systems, Tiruchengode, pp. 109-113, 2012.

[22] V. V. Singh, R. K. Jha and A. Ojha, "Significant region based robust watermarking scheme in lifting wavelet transform domain," Expert Systems with Applications, vol. 42, no. 21, pp. 8184-8197, 2015.

[23] V. V. Singh and R. K. Jha, "Improved watermarking technique based on significant difference of lifting wavelet coefficients," Signal, Image and Video Processing, vol. 9, no. 6, pp. 1443-1450, 2015.

[24] M. Farhan, D. Dangwal, S. Kumar and V. Malik, "A chaos-based image encryption algorithm based on multiresolution singular value decomposition and a symmetric attractor," Imaging Science Journal, vol. 68, no. 1, pp. 24-40, 2020.

[25] S. Siddharth, R. Singh and T. J. Siddiqui, "MSVD based image watermarking in NSCT domain," in 2016 Fourth Int. Conf. on Parallel, Distributed and Grid Computing, Waknaghat, India, IEEE, pp. 685-688, 2016.

[26] G. Efstratios, B. Philippe and A. H. Sameh, In Parallelism in Matrix Computations. Netherlands: Springer, 2016. [Online]. Available: https://www.springer.com/gp/book/9789401771870.

[27] Z. Yuhui, B. Jeon, D. Xu, Q. M. Wu and H. Zhang, "Image segmentation by generalized hierarchical fuzzy c-means algorithm," Journal of Intelligent \& Fuzzy Systems, vol. 28, no. 2, pp. 961-973, 2015. 
[28] H. Haseeb, A. K. Bashir, M. Ahmad, V. G. Menon, I. U. Afridi et al. "Real-time image dehazing by superpixels segmentation and guidance filter," Journal of Real-Time Image Processing, vol. 17, pp. 1-21, 2020.

[29] B. Jayant and A. Nayak, "Lifting wavelet transform based ultrasound image fusion scheme," in Adaptive Optics: Analysis, Methods \& Systems, Orlando, Florida, United States: Optical Society of America, pp. JTu4A-38, 2018.

[30] T. S. Sharmila and K. Ramar, "Efficient analysis of hybrid directional lifting technique for satellite image denoising," Signal Image Video Process, vol. 8, pp. 1399-1404, 2014.

[31] P. Jakub, G. Mikolajczak and J. Kowalski, "The use of the universal quality index for user recognition based on fingerprint analysis," in Multimedia and Network Information Systems, Springer: Cham, pp. 75-87, 2017.

[32] H. Haseeb, A. K. Bashir, R. Abbasi, W. Ahmad and B. Luo, "Single image defocus estimation by modified Gaussian function," Transactions on Emerging Telecommunications Technologies, vol. 30, no. 6, pp. e3611, 2019.

[33] A. E. Eddien, A. B. Hamza and P. Bhattacharya, "Improved image watermarking scheme using fast hadamard and discrete wavelet transforms," Journal of Electronic Imaging, vol. 16, no. 3, pp. 33020, 2007.

[34] T. Agung, and F. P. Permana, "Medical image watermarking with tamper detection and recovery using reversible watermarking with LSB modification and run length encoding (RLE) compression," in 2012 IEEE Int. Conf. on Communication, Networks and Satellite, Bali, Indonesia, IEEE, pp. 167-171, 2012. 